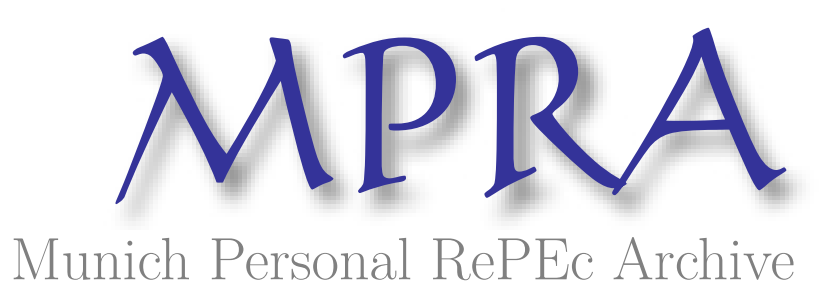

\title{
Minimization of Keane's Bump Function by the Repulsive Particle Swarm and the Differential Evolution Methods
}

\author{
Mishra, SK \\ North-Eastern Hill University, Shillong (India)
}

1 May 2007

Online at https://mpra.ub.uni-muenchen.de/3098/

MPRA Paper No. 3098, posted 05 May 2007 UTC 


\title{
Minimization of Keane's Bump Function by the Repulsive Particle Swarm and the Differential Evolution Methods
}

\author{
SK Mishra \\ Department of Economics \\ North-Eastern Hill University \\ Shillong (India)
}

I. Introduction: Andy Keane (1994) designed the "bump" function to test the performance of (constrained) optimization methods. The optimization problem of Keane's bump function may be presented (Keane, 1994) as follows:

$$
\text { Minimize } f(x)=-\left|\left\{\sum_{i=1}^{m} \cos ^{4}\left(x_{i}\right)-2 \prod_{i=1}^{m} \cos ^{2}\left(x_{i}\right)\right\} /\left(\sum_{i=1}^{m} i x_{i}^{2}\right)^{0.5}\right|
$$

subject to: $g_{1}(x): 0.75-\prod_{i=1}^{m} x_{i}<0 ; \quad g_{2}(x): \sum_{i=1}^{m} x_{i}-7.5 m<0 ; \quad 0<x_{i}<10$.

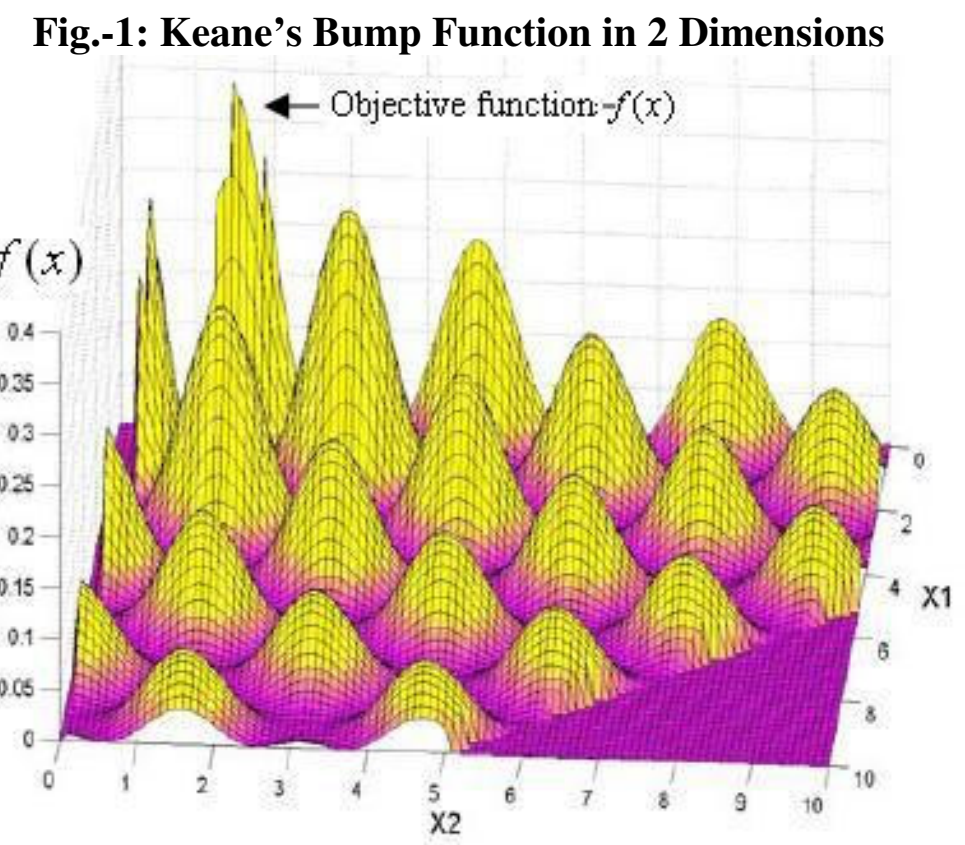

Source: Hacker, Eddy and Lewis (2002)
A visual appreciation of Keane's two-dimensional $(\mathrm{m}=2)$ bump function may be obtained from the graphical presentation (Fig.-1; Hacker et al., 2002). As the dimension (m) grows larger, the optimum value of the function becomes more and more difficult to obtain. Keane (1994) observed that for $m=20$ the value of $\min [f(x)]$ could be about -0.76 and for $m=50$ it could be about -0.835 but did not know this to be the case.

Keane's bump function is considered as a standard benchmark for nonlinear constrained optimization. It is highly multi-modal and its optimum is located at the nonlinear constrained boundary. Emmerich (2005, p. 116) noted that the true minimum of this function is unknown. Using their various hybridized Genetic Algorithms Hacker et al. (2002) obtained $\min [\mathrm{f}(\mathrm{x})]=-0.365$ for a 2-dimensional and -0.6737 for a 10 dimensional Keane's problem. They also found that the Genetic Algorithms (without hybridization) perform worse than their hybridized Genetic Algorithms.

The author is thankful to Dr. Kenneth L Judd of the Hoover Institution, Stanford University, USA for sending the paper of Hacker et al. (2002) that led to the present work on optimization of Keane's function, and his constructive suggestions to consider odd and oddly spaced dimensions, etc. 
II. The Objectives of this Paper: We intend in this paper to optimize Keane's function of different dimensions by the Repulsive Particle Swarm (RPS) and the Differential Evolution (DE) methods of global optimization. Our RPS is endowed with intensive local search ability. Similarly, our DE uses the most recent (available) formulation of crossover scheme suggested by Kenneth Price. We have developed our own computer programs in FORTRAN. Our programs have yielded very good results for quite varied and difficult problems (Mishra, 2006 (a, b \& c)). Programs are available on request* .

III. Results and Discussion: First we obtain $\min [\mathrm{f}(\mathrm{x})]$ for the two-dimensional $(\mathrm{m}=2)$ Keane's problem. We have DE $\min [(\mathrm{f})]=-0.364979746 ; \mathrm{g}_{1}(\mathrm{x})=-1.11022302 \mathrm{E}-016$ and $\mathrm{g}_{2}(\mathrm{x})=-11.9306415$ for $\mathrm{x}_{1}=1.60086044$ and $\mathrm{x}_{2}=0.468498055$. Against this, RPS $\min [(\mathrm{f})]=-0.364979123 ; \quad \mathrm{g}_{1}(\mathrm{x})=-3.82208509 \mathrm{E}-007 ; \mathrm{g}_{2}(\mathrm{x})=-11.9310703$ for $\mathrm{x}_{1}=$ 1.60025376 and $\mathrm{x}_{2}=0.468675907$. These results are comparable with the optimum value obtained by Hacker et al. The DE results are marginally better than the RPS results.

Emmerich (2005, p. 118) obtained about -0.6 as the minimum value of the $10-$ dimensional Keane problem. Hacker et al. report to have obtained $\min [\mathrm{f}(\mathrm{x})]=-0.6737$. We have obtained the DE $\min [(\mathrm{f})]=-0.747310362 ; \mathrm{g}_{1}(\mathrm{x})=-2.2849167 \mathrm{E}-011$ and $\mathrm{g}_{2}(\mathrm{x})$ $=-58.5724568$ for $\mathrm{x}$ given in Table-1(a). Against these we obtained by RPS method $\min [(\mathrm{f})]=-0.747309014 ; \mathrm{g}_{1}(\mathrm{x})=-4.64514816 \mathrm{E}-009 ;$ and $\mathrm{g}_{2}(\mathrm{x})=-58.5732418$ for $\mathrm{x}$ given in Table-1(b). Again, the DE performs better than the RPS.

Table-1(a). Values of Decision Variables of Keane $(m=10)$ obtained by DE

\begin{tabular}{|l|l|l|l|l|}
\hline 3.123889470 & 3.069156770 & 3.014282390 & 2.957588300 & 1.466044830 \\
\hline 0.368057943 & 0.363481530 & 0.359121133 & 0.354952823 & 0.350967972 \\
\hline
\end{tabular}

Table-1(b). Values of Decision Variables of Keane $(\mathrm{m}=10)$ obtained by RPS

\begin{tabular}{|l|l|l|l|l|}
\hline 3.124165150 & 3.068864800 & 3.015470120 & 2.955911380 & 1.465639690 \\
\hline 0.367340008 & 0.363605407 & 0.358758758 & 0.354977899 & 0.352024977 \\
\hline
\end{tabular}

For the 10-dimensional problem, evidently, our results are much better than those obtained by Hacker et al. by their hybridized Genetic Algorithm, which, in themselves are better than the (pure) Genetic algorithm results. They have not presented the values taken on by the decision variables (x). However, the values of $\mathrm{x}$ in our results indicate, first, that $x_{i}>x_{j} ; \forall j>i$. This particular observation is very important, although it is just a conjecture. A sub-optimal solution would not have such a sequence. Secondly, we conjecture that the values form two clusters with almost the same number of members.

For the 15-dimensional Keane's problem we have DE $\min [(\mathrm{f})]=-0.781647601$; $\mathrm{g}_{1}(\mathrm{x})=-5.18851406 \mathrm{E}-010 ; \mathrm{g}_{2}(\mathrm{x})=-88.5890866$. The decision variables take on the following values given in Table-2(a). Against these values of DE we have RPS min[(f)] $=-0.778452035 ; \mathrm{g}_{1}(\mathrm{x})=-1.42940888 \mathrm{E}-005 ; \mathrm{g}_{2}(\mathrm{x})=-88.6245989$ for $\mathrm{x}$ given in Table2(b) below. The RPS results are inferior to the DE results. The noted sequence is not given by the RPS results. The values of $x_{7}$ and $x_{8}$ have broken the said sequence. 


\begin{tabular}{|l|l|l|l|l|}
\hline \multicolumn{6}{|c|}{ Table-2(a). Values of Decision Variables of Keane $(\mathrm{m}=15)$ obtained by DE } \\
\hline 3.146293070 & 3.106145430 & 3.066581520 & 3.026848660 & 2.986415340 \\
\hline 2.944612650 & 1.432725200 & 0.414056148 & 0.409743392 & 0.405635026 \\
\hline 0.401726913 & 0.397869284 & 0.394212294 & 0.390652961 & 0.387395467 \\
\hline
\end{tabular}

Table-2(b). Values of Decision Variables of Keane $(\mathrm{m}=15)$ obtained by RPS

\begin{tabular}{|l|l|l|l|l|}
\hline 3.147885830 & 3.107285280 & 3.067863320 & 3.027769080 & 2.986310220 \\
\hline 2.947132220 & 0.418950619 & 1.373826510 & 0.413106309 & 0.406717883 \\
\hline 0.403270323 & 0.399151233 & 0.394427081 & 0.391056701 & 0.390648466 \\
\hline
\end{tabular}

For the 20-dimensional Keane's problem we have DE $\min [(\mathrm{f})]=-0.803619104$; $\mathrm{g}_{1}(\mathrm{x})=-4.95659069 \mathrm{E}-012 ; \mathrm{g}_{2}(\mathrm{x})=-119.067416$. The decision variables take on the following values given in Table-3(a). We obtain the RPS $\min [(\mathrm{f})]=-0.785263489 ; \mathrm{g}_{1}(\mathrm{x})$ $=-2.13415866 \mathrm{E}-005 ; \mathrm{g}_{2}(\mathrm{x})=-117.548076$ for the values of $\mathrm{x}$ given in Table-3(b).

Table-3(a). Values of Decision Variables of Keane $(\mathrm{m}=20)$ obtained by DE

\begin{tabular}{|l|l|l|l|l|}
\hline 3.162462120 & 3.128331570 & 3.094791700 & 3.061449390 & 3.027931110 \\
\hline 2.993829330 & 2.958666990 & 2.921839070 & 0.494829273 & 0.488358755 \\
\hline 0.482312620 & 0.476648099 & 0.471293545 & 0.466228022 & 0.461417418 \\
\hline 0.456836629 & 0.452460752 & 0.448267681 & 0.444248266 & 0.440381976 \\
\hline
\end{tabular}

Table-3(b). Values of Decision Variables of Keane $(\mathrm{m}=20)$ obtained by RPS

\begin{tabular}{|l|l|l|l|l|}
\hline 3.151484020 & 3.119328890 & 3.086598240 & 3.053529180 & 3.021145250 \\
\hline 2.985534770 & 2.949188660 & 2.911211960 & 0.418488616 & 2.821296710 \\
\hline 0.410584741 & 0.405855243 & 0.399953254 & 0.398307630 & 0.394483277 \\
\hline 0.391179683 & 0.388534529 & 0.383874458 & 0.382995909 & 0.378349313 \\
\hline
\end{tabular}

We note that the DE results obey the observed rule of sequence while the RPS results, which are sub-optimal, do not obey the said rule. We also note that while Keane (1994) observed that for $\mathrm{m}=20$ the value of $\min [\mathrm{f}(\mathrm{x})]$ could be about -0.76 , we obtain $\mathrm{DE}$ $\min [(\mathrm{f})]=-0.803619104$. This result is surely better than the one envisaged by Keane. However, Ong and Keane (2003, p. 12) and Ong et al. (2005 ?) mention that the minimal value obtained by them is approximately -0.81 . If it is so, we have not been able to obtain the minimum value of the function. Keane in his personal letter (email dated 4.5.2007) informed the author that the best value for $\mathrm{m}=20$ known to him till date is -0.803619104 .

\begin{tabular}{|l|l|l|l|l|}
\hline \multicolumn{5}{|c|}{ Table-4. Values of Decision Variables of Keane $(\mathrm{m}=30)$ obtained by DE } \\
\hline 3.168225530 & 3.146211650 & 3.124531090 & 3.102979710 & 3.081823620 \\
\hline 3.060544800 & 3.039195590 & 3.017679840 & 2.995636850 & 2.973543750 \\
\hline 2.950666480 & 2.927562460 & 2.903088710 & 0.440434895 & 0.437505191 \\
\hline 0.435103340 & 0.432460693 & 0.429815709 & 0.427295405 & 0.424698735 \\
\hline 0.422641158 & 0.420028735 & 0.417678117 & 0.415577752 & 0.413108742 \\
\hline 0.410869231 & 0.408999549 & 0.406826514 & 0.405042008 & 0.402869708 \\
\hline
\end{tabular}


For the 30-dimensional Keane's problem we have DE $\min [(\mathrm{f})]=-0.818056222$; $\mathrm{g}_{1}(\mathrm{x})=-1.90829275 \mathrm{E}-009 ; \mathrm{g}_{2}(\mathrm{x})=-177.357354$. The decision variables take on the values given in Table-4. The RPS results are grossly sub-optimal and hence we do not present them.

For the 40-dimensional Keane's problem we have DE $\min [(\mathrm{f})]=-0.826624404$; $\mathrm{g}_{1}(\mathrm{x})=-4.67459549 \mathrm{E}-009 ; \mathrm{g}_{2}(\mathrm{x})=-237.241084$. The decision variables take on the following values given in Table-5. The RPS results are grossly sub-optimal and hence we do not present them.

\begin{tabular}{|l|l|l|l|l|}
\hline \multicolumn{5}{|c|}{ Table-5. Values of Decision Variables of Keane $(\mathrm{m}=40)$ obtained by DE } \\
\hline 3.176402220 & 3.159781680 & 3.143267960 & 3.126970650 & 3.110958340 \\
\hline 3.094833420 & 3.078715320 & 3.062493030 & 3.046925820 & 3.030744620 \\
\hline 3.014489370 & 2.998191690 & 2.981676650 & 2.964750240 & 2.947671240 \\
\hline 2.930427940 & 2.912463350 & 0.455727042 & 0.453400834 & 0.451294809 \\
\hline 0.448984473 & 0.446911098 & 0.444660768 & 0.442769918 & 0.440588651 \\
\hline 0.438735980 & 0.436742512 & 0.435131699 & 0.433072143 & 0.431025920 \\
\hline 0.429591143 & 0.427762652 & 0.425920239 & 0.424344581 & 0.422578191 \\
\hline 0.420945959 & 0.419335333 & 0.417769877 & 0.416132722 & 0.414726294 \\
\hline
\end{tabular}

Optimization of the 50-dimensional Keane's problem was problematic. We had to do some fine-tuning of the DE parameters and some trial and error too. Finally, [for $\mathrm{RX} 1=0.5, \mathrm{RX} 2=0.7$ and $\mathrm{F}=0.05$ : these are detailed out in the DE program written by us] we have DE $\min [(\mathrm{f})]=-0.83078783 ; \mathrm{g}_{1}(\mathrm{x})=-2.55134022 \mathrm{E}-008 ; \mathrm{g}_{2}(\mathrm{x})=-297.149824$. The decision variables take on the following values given in Table-6. The RPS results are grossly sub-optimal and hence we do not present them

\begin{tabular}{|l|l|l|l|l|}
\hline \multicolumn{5}{|c|}{ Table-6. Values of Decision Variables of Keane $(\mathrm{m}=50)$ obtained by DE } \\
\hline 2.984331850 & 2.970850220 & 2.957100110 & 2.943168300 & 2.929067060 \\
\hline 2.914580720 & 0.465266845 & 0.463396171 & 0.461448453 & 0.459610933 \\
\hline 0.457759997 & 0.456007425 & 0.454318803 & 0.452430869 & 0.450861344 \\
\hline 0.449163331 & 0.447302715 & 0.445867968 & 0.444297375 & 0.442654703 \\
\hline 0.440993800 & 0.439496353 & 0.438148497 & 0.436730303 & 0.435083505 \\
\hline 0.433749816 & 0.432327620 & 0.430812420 & 0.429525278 & 0.428145716 \\
\hline 0.426877925 & 0.425525920 & 0.424203890 & 0.422892481 & 0.421473178 \\
\hline 2.984331850 & 2.970850220 & 2.957100110 & 2.943168300 & 2.929067060 \\
\hline 2.914580720 & 0.465266845 & 0.463396171 & 0.461448453 & 0.459610933 \\
\hline 0.457759997 & 0.456007425 & 0.454318803 & 0.452430869 & 0.450861344 \\
\hline
\end{tabular}

Keane (1994) mentioned that for $m=50 \min [(f)]$ could be around -0.835 . The number obtained by us is -0.83078783 (and the decision variables satisfy the sequence noted earlier). We cannot claim that the sequence conjectured by us provides the necessary and sufficient condition for optimality of values taken on by the decision variables, although the condition appears to be necessary. Keane in his personal letter (email dated 4.5.2007) informed the author that the best value known to him for $m=50$ till date is: -0.835262348 . 
IV. A Useful Application of Our Conjecture: Now we make an attempt to (possibly) take advantage of our conjecture on the pattern exhibited by the values of decision variable so far. That is: $x_{i}>x_{j} ; \forall j>i$. Before every function evaluation we arrange the values of the decision variables in a descending order and then evaluate the Keane's function (and the constraints). We find, to our pleasant surprise, that it works well and gives a new minimum (for $m=50$ ), which is very close to the value $(\simeq-0.835)$ envisaged by Keane. Now, after incorporating our conjecture in the computation, we obtain: DE $\min [(\mathrm{f})]=\mathbf{- 0 . 8 3 4 9 8 5 1 2 6}$ and $\mathrm{g}_{1}(\mathrm{x})=-9.86513138 \mathrm{E}-009 ; \mathrm{g}_{2}(\mathrm{x})=-294.382754$. The decision variables take on the values given in Table-7. The RPS results are close to the DE results, but sub-optimal. The RPS $\min [(\mathrm{f})]=-0.83181972 ; \mathrm{g}_{1}(\mathrm{x})=-3.97261395 \mathrm{E}-05$; $\mathrm{g}_{2}(\mathrm{x})=-292.704914$.

\begin{tabular}{|l|l|l|l|l|}
\hline \multicolumn{5}{|c|}{ Table-7. Values of Decision Variables of Keane $(\mathrm{m}=50)$ obtained by DE } \\
\hline 6.281881470 & 3.165864960 & 3.152419640 & 3.139117420 & 3.125910180 \\
\hline 3.112937380 & 3.099820120 & 3.086810400 & 3.073914590 & 3.060912370 \\
\hline 3.047946650 & 3.034998900 & 3.021821370 & 3.008627600 & 2.995345870 \\
\hline 2.982032700 & 2.968429500 & 2.954671080 & 2.940647550 & 2.926380720 \\
\hline 2.911877500 & 0.454055821 & 0.452282938 & 0.450327194 & 0.448694221 \\
\hline 0.446908360 & 0.445253680 & 0.443532095 & 0.441920750 & 0.440236963 \\
\hline 0.438736885 & 0.437136832 & 0.435642454 & 0.434122273 & 0.432614935 \\
\hline 0.431118544 & 0.429681262 & 0.428261041 & 0.426880934 & 0.425427247 \\
\hline 0.424099015 & 0.422711491 & 0.421441660 & 0.420066322 & 0.418684131 \\
\hline 0.417438227 & 0.416297458 & 0.415067783 & 0.413740624 & 0.412497031 \\
\hline
\end{tabular}

It has been mentioned earlier that Ong and Keane (2003, p. 12) and Ong et al. (2005 ?) report that the minimal value obtained by them is approximately -0.81 (for $\mathrm{m}=20$ ). With the success experience due to incorporating our conjecture (in case of $\mathrm{m}=50$ ) we are tempted to re-compute the $\min [(\mathrm{f})]$ for $\mathrm{m}=20$. However, we have not been able to obtain any better results (than -0.803619104 , reported earlier).

For $\mathrm{m}=60$, we obtain DE $\min [(\mathrm{f})]=-0.835835669 ; \mathrm{g}_{1}(\mathrm{x})=-6.7704109 \mathrm{E}-010$; $\mathrm{g}_{2}(\mathrm{x})=-352.652189$. The decision variables take on the following values given in Table8(a). In this case, however, the RPS results are better than the DE results. We obtain RPS $\min [(\mathrm{f})]=-0.837746743$. The values of the decision variables are given in Table-8(b).

\begin{tabular}{|l|l|l|l|l|l|}
\hline \multicolumn{6}{|c|}{ Table-8(a). Values of Decision Variables of Keane $(\mathrm{m}=60)$ obtained by DE } \\
\hline 6.285338790 & 3.167861920 & 3.156781730 & 3.145809150 & 3.134964570 & 3.124223950 \\
\hline 3.113376380 & 3.102748600 & 3.092086930 & 3.081489920 & 3.070871930 & 3.060171490 \\
\hline 3.049418270 & 3.038716460 & 3.028148500 & 3.017316210 & 3.006609820 & 2.995700460 \\
\hline 2.984574080 & 2.973620460 & 2.962402510 & 2.950981780 & 2.939532730 & 2.927839980 \\
\hline 2.915972240 & 2.903798910 & 0.434815891 & 0.433294979 & 0.432069993 & 0.430621744 \\
\hline 0.429421427 & 0.427964211 & 0.426917113 & 0.425579719 & 0.424408800 & 0.423234386 \\
\hline 0.422015631 & 0.420993818 & 0.419629141 & 0.418482726 & 0.417330303 & 0.416204475 \\
\hline 0.415108638 & 0.414248869 & 0.413012358 & 0.411953992 & 0.410849798 & 0.409916159 \\
\hline 0.408821128 & 0.407824210 & 0.406679669 & 0.405756013 & 0.404700677 & 0.403723423 \\
\hline 0.402652061 & 0.401793732 & 0.400705123 & 0.399886726 & 0.398889977 & 0.397946680 \\
\hline
\end{tabular}




\begin{tabular}{|l|l|l|l|l|l|}
\hline \multicolumn{6}{|c|}{ Table-8(b). Values of Decision Variables of Keane $(\mathrm{m}=60)$ obtained by RPS } \\
\hline 6.286843320 & 3.173492270 & 3.160683060 & 3.149693970 & 3.138712600 & 3.126625110 \\
\hline 3.115790130 & 3.105019480 & 3.093778840 & 3.080946500 & 3.071653830 & 3.061515640 \\
\hline 3.049746000 & 3.036256360 & 3.027641820 & 3.017186050 & 3.006125490 & 2.996929890 \\
\hline 2.982453710 & 2.971695830 & 2.959466880 & 2.951034220 & 2.939888170 & 2.925757420 \\
\hline 2.910715510 & 0.463715298 & 0.462422111 & 0.457899571 & 0.457513834 & 0.456935488 \\
\hline 0.454491776 & 0.452602034 & 0.451457866 & 0.451053248 & 0.447671899 & 0.446884213 \\
\hline 0.445433219 & 0.444898576 & 0.444218376 & 0.441387240 & 0.440728984 & 0.440399367 \\
\hline 0.438915986 & 0.438476776 & 0.436563227 & 0.435000188 & 0.433114197 & 0.432494583 \\
\hline 0.430914958 & 0.429462320 & 0.428974897 & 0.427472923 & 0.426076003 & 0.424951615 \\
\hline 0.423772714 & 0.423230527 & 0.420626442 & 0.419746824 & 0.418076501 & 0.415879864 \\
\hline
\end{tabular}

Finally we have run DE to optimize the Keane function for $m=100$. We would also like to mention that we used 4321 as the initial seed for generating the random numbers; population size $=1000$; pcross $=0.9$; fact $=0.05$ and 5677 as the second random number seed in the DE subroutine. These inputs/parameters are defined in our computer program. We also used our conjecture to arrange $\mathrm{x}$ in a descending order We obtained $\mathrm{DE} \min [(\mathrm{f})]=-0.844219651 ; \mathrm{g}_{1}(\mathrm{x})=-2.51972313 \mathrm{E}-008 ; \mathrm{g}_{2}(\mathrm{x})=-586.659534$. Values of $\mathrm{x}$ are presented in Table-9(a) below.

\begin{tabular}{|l|l|l|l|l|}
\hline \multicolumn{5}{|c|}{ Table-9(a). Values of Decision Variables of Keane $(\mathrm{m}=100)$ obtained by DE } \\
\hline 9.421209850 & 6.281568910 & 3.172221960 & 3.165330690 & 3.158407070 \\
\hline 3.151790900 & 3.144986000 & 3.138420350 & 3.131239320 & 3.124581260 \\
\hline 3.118033120 & 3.111275770 & 3.104712750 & 3.098148350 & 3.091554370 \\
\hline 3.084750280 & 3.078285450 & 3.071863590 & 3.065027900 & 3.058509040 \\
\hline 3.051894330 & 3.045239320 & 3.038673780 & 3.031942040 & 3.025294780 \\
\hline 3.018717050 & 3.011990360 & 3.005180740 & 2.998547060 & 2.991581570 \\
\hline 2.984878120 & 2.978015990 & 2.971031220 & 2.964043880 & 2.956997030 \\
\hline 2.950130590 & 2.942822710 & 2.935704570 & 2.928359500 & 2.920978890 \\
\hline 2.913649490 & 2.906208780 & 0.453854241 & 0.452817124 & 0.452100592 \\
\hline 0.451067421 & 0.450422860 & 0.449256343 & 0.448279024 & 0.447387629 \\
\hline 0.446720503 & 0.445666150 & 0.444731075 & 0.444080540 & 0.443086457 \\
\hline 0.442474034 & 0.441448740 & 0.440789329 & 0.439877311 & 0.439219450 \\
\hline 0.438206790 & 0.437293937 & 0.436692347 & 0.435877182 & 0.435154888 \\
\hline 0.434306103 & 0.433404136 & 0.432766335 & 0.431938810 & 0.431135854 \\
\hline 0.430542180 & 0.429982791 & 0.428823204 & 0.428462627 & 0.427640123 \\
\hline 0.427102876 & 0.426255391 & 0.425483535 & 0.424786864 & 0.424002049 \\
\hline 0.423462434 & 0.422801656 & 0.422098679 & 0.421377911 & 0.420720915 \\
\hline 0.420010667 & 0.419362836 & 0.418770729 & 0.418116471 & 0.417478448 \\
\hline 0.416845509 & 0.416097790 & 0.415462624 & 0.414916930 & 0.414249639 \\
\hline 0.413555041 & 0.412891312 & 0.412286270 & 0.411823787 & 0.411201187 \\
\hline
\end{tabular}

We have run the RPS with random seed $=7337$. The RPS $\min [(\mathrm{f})]=-0.84246233$; $\mathrm{g}_{1}(\mathrm{x})=-0.000523752645 ; \mathrm{g}_{2}(\mathrm{x})=-587.939518$, which is inferior to the DE $\min [(\mathrm{f})]$. The values of $\mathrm{x}$ are presented in Table-9(b). It appears that arranging the values of decision variables in descending order affects the performance of the DE as well as the RPS unpredictably, although it is difficult to generalize such an observation. Further, we do not claim that for $m=100$ our DE results are the best, although one may note that Liu and Lewis (2002) obtained -0.84421 and -0.8448539 for $\mathrm{m}=200$ and $\mathrm{m}=1000$ respectively. 
Our results for $\mathrm{m}=100(\min (\mathrm{f})=-0.844219651)$ is smaller than Liu-Lewis' -0.84421 for $\mathrm{m}=200$, which is anomalous. Thus, Liu-Lewis' $\min (f)$ for $m=200$ is grossly sub-optimal.

\begin{tabular}{|r|r|r|r|r|}
\hline \multicolumn{5}{|c|}{ Table-9(b). Values of Decision Variables of Keane $(\mathrm{m}=100)$ obtained by RPS } \\
\hline 6.295905660 & 6.283013840 & 3.170384370 & 3.165686900 & 3.157958600 \\
\hline 3.151463240 & 3.142404470 & 3.139385180 & 3.125719650 & 3.121996200 \\
\hline 3.114943680 & 3.111719990 & 3.102821160 & 3.098702340 & 3.091716510 \\
\hline 3.088209640 & 3.074270960 & 3.071114580 & 3.064350170 & 3.054508540 \\
\hline 3.054326000 & 3.049031790 & 3.039193270 & 3.030651010 & 3.027172050 \\
\hline 3.021449410 & 3.010442630 & 3.010060930 & 2.996908920 & 2.994202160 \\
\hline 2.985068020 & 2.977596560 & 2.971414430 & 2.968615030 & 2.960406750 \\
\hline 2.954659140 & 2.943754670 & 2.936470690 & 2.928697320 & 2.928315880 \\
\hline 2.915114150 & 2.903913760 & 2.903039740 & 0.440651023 & 0.439306900 \\
\hline 0.439129498 & 0.438640185 & 0.437377084 & 0.436875468 & 0.436572276 \\
\hline 0.435786420 & 0.435199692 & 0.433733227 & 0.432524090 & 0.431707741 \\
\hline 0.430846454 & 0.430573021 & 0.430461588 & 0.430113172 & 0.428261855 \\
\hline 0.428255325 & 0.426487033 & 0.424873853 & 0.424032799 & 0.423253553 \\
\hline 0.423011175 & 0.423006205 & 0.421760827 & 0.421633427 & 0.420970330 \\
\hline 0.419523842 & 0.418669684 & 0.418180749 & 0.417961379 & 0.417258479 \\
\hline 0.417211605 & 0.416822491 & 0.415255666 & 0.414534559 & 0.414523647 \\
\hline 0.414407966 & 0.414029761 & 0.412823195 & 0.411574032 & 0.410124136 \\
\hline 0.409683366 & 0.409510891 & 0.408945793 & 0.407934372 & 0.406994044 \\
\hline 0.406725228 & 0.405931032 & 0.405341784 & 0.404075017 & 0.403745876 \\
\hline 0.403488194 & 0.400118948 & 0.399434791 & 0.397844945 & 0.395982412 \\
\hline
\end{tabular}

V. Minimization of Keane Function with Odd Number of Variables: Do the properties of Keane problem depend on whether its dimension is odd or oddly chosen? We have (arbitrarily) chosen some odd numbers; 17, 23, 27, 35, 43 and 47 to study the behavior of Keane function. We have ignored small dimensions like 3, 7, etc. For $m=5$ we have DE $\min \left[(\mathrm{f})=-0.634448687, \mathrm{~g}_{1}(\mathrm{x})=-4.4408921 \mathrm{E}-016, \mathrm{~g}_{2}(\mathrm{x})=-28.4863914\right.$ for $\mathrm{x}$ $=(3.07581932,2.99199522,1.47579373,0.236691358,0.233308996)$. A perusal of the following tables - $10(\mathrm{a}, \mathrm{b})$ through $15(\mathrm{a}, \mathrm{b})$ - reveals that it is not so. Hence, we conclude that the minimum values of the problem monotonously decline with the increase in the dimension of Keane problem.

Table-10(a). Values of Decision Variables of Keane $(\mathrm{m}=17)$ obtained by DE $\operatorname{Min}[\mathrm{f}(\mathrm{x})]=-0.79150563 ; \mathrm{g}_{1}(\mathrm{x})=-3.85410481 \mathrm{E}-010 ; \mathrm{g}_{2}(\mathrm{x})=-100.81833$

\begin{tabular}{|l|l|l|l|l|}
\hline 3.151991990 & 3.111505760 & 3.071640430 & 3.031343620 & 2.990185690 \\
\hline 2.947234280 & 2.900955860 & 0.475303034 & 0.468048135 & 0.461194008 \\
\hline 0.454953916 & 0.449055912 & 0.443555158 & 0.438405917 & 0.433369424 \\
\hline 0.428717289 & 0.424209287 & & & \\
\hline
\end{tabular}

\begin{tabular}{|l|l|l|l|l|}
\hline \multicolumn{5}{|c|}{$\begin{array}{r}\text { Table-10(b). Values of Decision Variables of Keane }(\mathrm{m}=17) \text { obtained by RPS } \\
\text { Min[ }[\mathrm{f}(\mathrm{x})]=-0.791503305 ; \mathrm{g}_{1}(\mathrm{x})=-6.14730171 \mathrm{E}-006 ; \mathrm{g}_{2}(\mathrm{x})=-100.813254\end{array}$} \\
\hline 3.153072390 & 3.110523170 & 3.074380460 & 3.030577260 & 2.990643470 \\
\hline 2.948230310 & 2.903373010 & 0.475140236 & 0.468015020 & 0.461406996 \\
\hline 0.455065868 & 0.448695305 & 0.443058078 & 0.438121089 & 0.434204415 \\
\hline 0.428833184 & 0.423405586 & & & \\
\hline
\end{tabular}


Table-11(a). Values of Decision Variables of Keane $(m=23)$ obtained by DE $\operatorname{Min}[\mathrm{f}(\mathrm{x})]=-0.810036554 ; \mathrm{g}_{1}(\mathrm{x})=-4.29345448 \mathrm{E}-012 ; \mathrm{g}_{2}(\mathrm{x})=-137.329364$

\begin{tabular}{|l|l|l|l|l|}
\hline 3.169694760 & 3.139982510 & 3.110819230 & 3.081854680 & 3.053000050 \\
\hline 3.023921240 & 2.994482270 & 2.964326540 & 2.933023260 & 0.510288829 \\
\hline 0.504420618 & 0.498649275 & 0.493512848 & 0.488546279 & 0.483523272 \\
\hline 0.479153946 & 0.474755611 & 0.470648484 & 0.466631399 & 0.462846694 \\
\hline 0.459054987 & 0.455553637 & 0.451945688 & & \\
\hline
\end{tabular}

Table-11(b). Values of Decision Variables of Keane $(\mathrm{m}=23)$ obtained by RPS $\operatorname{Min}[\mathrm{f}(\mathrm{x})]=-0.806443992 ; \mathrm{g}_{1}(\mathrm{x})=-2.44754988 \mathrm{E}-005 ; \mathrm{g}_{2}(\mathrm{x})=-135.786281$

\begin{tabular}{|l|l|l|l|l|}
\hline 3.160189530 & 3.132605360 & 3.101283350 & 3.073486860 & 3.045908980 \\
\hline 3.016721420 & 2.984253490 & 2.957205140 & 2.925348010 & 2.891203450 \\
\hline 0.437316502 & 0.434618030 & 0.429887890 & 0.427719236 & 0.422809225 \\
\hline 0.421139133 & 0.416725599 & 0.413117438 & 0.409371097 & 0.407704275 \\
\hline 0.405107257 & 0.401105071 & 0.398892942 & & \\
\hline
\end{tabular}

Table-12(a). Values of Decision Variables of Keane $(\mathrm{m}=27)$ obtained by DE $\operatorname{Min}[\mathrm{f}(\mathrm{x})]=-0.811434228 ; \mathrm{g}_{1}(\mathrm{x})=-2.53111421 \mathrm{E}-010 ; \mathrm{g}_{2}(\mathrm{x})=-159.083939$

\begin{tabular}{|l|l|l|l|l|}
\hline 3.162640070 & 3.138297520 & 3.114299000 & 3.090456170 & 3.066700220 \\
\hline 3.042930870 & 3.018928310 & 2.994522010 & 2.969607880 & 2.943953290 \\
\hline 2.917228550 & 2.889110810 & 0.422657874 & 0.419707697 & 0.416940414 \\
\hline 0.414145420 & 0.411515639 & 0.408872113 & 0.406368134 & 0.403919424 \\
\hline 0.401489671 & 0.399182553 & 0.396883387 & 0.394646104 & 0.392482705 \\
\hline 0.390330937 & 0.388244176 & & & \\
\hline
\end{tabular}

Table-12(b). Values of Decision Variables of Keane $(\mathrm{m}=27)$ obtained by RPS $\operatorname{Min}[\mathrm{f}(\mathrm{x})]=-0.816943304 ; \mathrm{g}_{1}(\mathrm{x})=-1.19269072 \mathrm{E}-005 ; \mathrm{g}_{2}(\mathrm{x})=-160.653353$

\begin{tabular}{|l|l|l|l|l|}
\hline 3.173409950 & 3.148240590 & 3.122005090 & 3.099207630 & 3.073407570 \\
\hline 3.050460980 & 3.024861520 & 3.000385800 & 2.975618330 & 2.950741980 \\
\hline 2.920317690 & 0.483482816 & 0.477704153 & 0.475182525 & 0.471337525 \\
\hline 0.467439896 & 0.464773078 & 0.461536009 & 0.459642008 & 0.454704367 \\
\hline 0.450330010 & 0.447936313 & 0.444572224 & 0.440030300 & 0.438913875 \\
\hline 0.436688512 & 0.433716710 & & & \\
\hline
\end{tabular}

Table-13(a). Values of Decision Variables of Keane $(m=35)$ obtained by DE $\operatorname{Min}[\mathrm{f}(\mathrm{x})]=-0.823249408 ; \mathrm{g}_{1}(\mathrm{x})=-2.19188578 \mathrm{E}-009 ; \mathrm{g}_{2}(\mathrm{x})=-207.294331$

\begin{tabular}{|l|l|l|l|l|}
\hline 3.173022940 & 3.154137450 & 3.135351760 & 3.116948540 & 3.098632110 \\
\hline 3.080316430 & 3.062019850 & 3.043838080 & 3.025530650 & 3.007087470 \\
\hline 2.988219760 & 2.969192990 & 2.949682640 & 2.929865560 & 2.909301360 \\
\hline 0.4490874411 & 0.446489526 & 0.443976814 & 0.441598501 & 0.439260019 \\
\hline 0.437147128 & 0.434902785 & 0.432761739 & 0.430545870 & 0.428373333 \\
\hline 0.426403086 & 0.424338982 & 0.422526037 & 0.420436699 & 0.418566482 \\
\hline 0.417004278 & 0.414856581 & 0.413117846 & 0.411460307 & 0.409667854 \\
\hline
\end{tabular}




\begin{tabular}{|c|c|c|c|c|}
\hline \multicolumn{3}{|c|}{$\begin{array}{c}\text { Table-13(b). Values of Decision Variables of Keane }(\mathrm{m}=35) \text { obtained by RPS } \\
\text { Min[f( }(\mathrm{x})]=-0.825277297\end{array} \mathrm{~g}_{1}(\mathrm{x})=-0.000119139164 ; \mathrm{g}_{2}(\mathrm{x})=-204.505815$} \\
\hline 6.269726250 & 3.154360330 & 3.133103100 & 3.114955030 & 3.098820910 \\
\hline 3.079824170 & 3.060554440 & 3.044914760 & 3.023623240 & 3.005605400 \\
\hline 2.985462770 & 2.965903150 & 2.946515240 & 2.925727920 & 2.905643010 \\
\hline 0.433505755 & 0.431011707 & 0.428275671 & 0.426509492 & 0.424762597 \\
\hline 0.422812865 & 0.420239079 & 0.419822901 & 0.418930795 & 0.413752342 \\
\hline 0.410895308 & 0.410283080 & 0.408714840 & 0.408045066 & 0.405630103 \\
\hline 0.404382321 & 0.402369030 & 0.400914304 & 0.397253255 & 0.391334432 \\
\hline
\end{tabular}

Table-14(a). Values of Decision Variables of Keane $(m=43)$ obtained by DE $\operatorname{Min}[\mathrm{f}(\mathrm{x})]=-0.83226135 ; \mathrm{g}_{1}(\mathrm{x})=-8.43495951 \mathrm{E}-009 ; \mathrm{g}_{2}(\mathrm{x})=-254.377563$

\begin{tabular}{|l|l|l|l|l|}
\hline 6.279437570 & 3.166025720 & 3.149944230 & 3.134193120 & 3.118384230 \\
\hline 3.102904180 & 3.087267970 & 3.071788540 & 3.056379620 & 3.040696730 \\
\hline 3.024949450 & 3.009350790 & 2.993456050 & 2.977385710 & 2.961086450 \\
\hline 2.944514810 & 2.927548890 & 0.493388747 & 0.490601423 & 0.487922748 \\
\hline 0.485142273 & 0.482630155 & 0.480188053 & 0.477827961 & 0.475449255 \\
\hline 0.473114249 & 0.470720458 & 0.468456669 & 0.466343132 & 0.464350740 \\
\hline 0.462260514 & 0.460134599 & 0.458287613 & 0.456369695 & 0.454410171 \\
\hline 0.452403380 & 0.450637388 & 0.448870370 & 0.446976988 & 0.445250201 \\
\hline 0.443408596 & 0.441789263 & 0.440188104 & & \\
\hline
\end{tabular}

Table-14(b). Values of Decision Variables of Keane $(m=43)$ obtained by RPS $\operatorname{Min}[\mathrm{f}(\mathrm{x})]=-0.832259899 ; \mathrm{g}_{1}(\mathrm{x})=-6.7151146 \mathrm{E}-005 ; \mathrm{g}_{2}(\mathrm{x})=-252.749098$

\begin{tabular}{|l|l|l|l|l|}
\hline 6.281229680 & 3.158936610 & 3.148112900 & 3.134537550 & 3.118666760 \\
\hline 3.099927430 & 3.086366350 & 3.070651230 & 3.056637670 & 3.041661580 \\
\hline 3.025227620 & 3.011444720 & 2.994136920 & 2.979632390 & 2.963655390 \\
\hline 2.945970420 & 2.931033600 & 2.915054880 & 0.455455069 & 0.451050569 \\
\hline 0.449413031 & 0.447793497 & 0.444416653 & 0.443580846 & 0.442544677 \\
\hline 0.440354860 & 0.436806643 & 0.436328404 & 0.434712866 & 0.431599120 \\
\hline 0.431060144 & 0.429201676 & 0.428003131 & 0.426853958 & 0.426739990 \\
\hline 0.423883701 & 0.420301219 & 0.418990143 & 0.417206138 & 0.415803124 \\
\hline 0.412705272 & 0.411980526 & 0.411233373 & & \\
\hline
\end{tabular}

Table-15(a). Values of Decision Variables of Keane $(\mathrm{m}=47)$ obtained by DE $\operatorname{Min}[\mathrm{f}(\mathrm{x})]=-0.833300903 ; \mathrm{g}_{1}(\mathrm{x})=-5.64001357 \mathrm{E}-009 ; \mathrm{g}_{2}(\mathrm{x})=-276.077314$

\begin{tabular}{|l|l|l|l|l|}
\hline 6.279663750 & 3.162847070 & 3.148694640 & 3.134779200 & 3.120871690 \\
\hline 3.107283320 & 3.093539880 & 3.079848380 & 3.066213940 & 3.052490340 \\
\hline 3.038756200 & 3.025031050 & 3.011176820 & 2.997157170 & 2.983039540 \\
\hline 2.968763490 & 2.954266890 & 2.939472010 & 2.924405520 & 2.909122960 \\
\hline 0.443783424 & 0.441847883 & 0.440139288 & 0.438398466 & 0.436734371 \\
\hline 0.435098197 & 0.433369075 & 0.431822240 & 0.430138948 & 0.428540952 \\
\hline 0.426973748 & 0.425434393 & 0.423966985 & 0.422492736 & 0.421050529 \\
\hline 0.419561255 & 0.418206208 & 0.416749228 & 0.415372910 & 0.413967574 \\
\hline 0.412681692 & 0.411387253 & 0.410076023 & 0.408800246 & 0.407530631 \\
\hline 0.406203464 & 0.404934000 & & & \\
\hline
\end{tabular}




\begin{tabular}{|c|c|c|c|c|}
\hline \multicolumn{6}{|c|}{ Table-15(b). Values of Decision Variables of Keane $(\mathrm{m}=47)$ obtained by RPS } \\
Min[f(x)]= -0.82892792; $\mathrm{g}_{1}(\mathrm{x})=-5.64914444 \mathrm{E}-005 ; \mathrm{g}_{2}(\mathrm{x})=-274.399424$ \\
\hline 6.280865570 & 3.156188810 & 3.146774090 & 3.131048300 & 3.117603860 \\
\hline 3.105551320 & 3.089657260 & 3.079060790 & 3.064843410 & 3.046563230 \\
\hline 3.040863700 & 3.023017970 & 3.012707150 & 2.997817240 & 2.983747000 \\
\hline 2.970102890 & 2.956072610 & 2.940757960 & 2.926886930 & 2.911972030 \\
\hline 2.899856250 & 0.410299283 & 0.408159859 & 0.407157204 & 0.405598548 \\
\hline 0.403227902 & 0.402294096 & 0.402016870 & 0.400016782 & 0.399578215 \\
\hline 0.398264780 & 0.397064629 & 0.395442428 & 0.393422846 & 0.392503227 \\
\hline 0.392036651 & 0.390980169 & 0.390029983 & 0.388256750 & 0.386166054 \\
\hline 0.384685340 & 0.383143291 & 0.381729759 & 0.380716239 & 0.376113897 \\
\hline 0.375371828 & 0.374340769 & & & \\
\hline
\end{tabular}

VI. Conclusion: A not-so-exhaustive survey of literature on optimization of Keane's function suggests us that many researchers avoid mentioning the values of objective function, constraints and decision variables that they obtained in their works. They

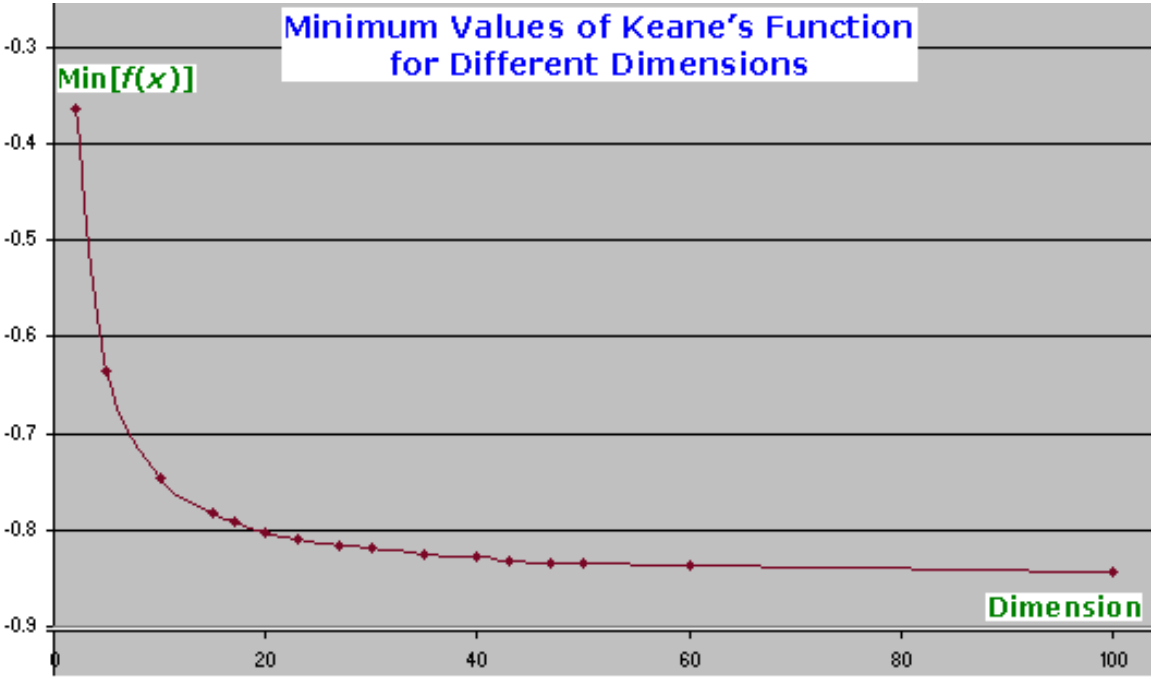
mention that the program, method or algorithm used by them was repeated so-andso many times. However, they have hesitated to mention the range - the upper and the lower limits within which they had obtained their results. Measures like mean, median or standard deviation only blur the findings and perhaps conceal much more than they reveal. If Emmerich (2005) is right in stating that the true minima of Keane's function for different dimensions are unknown, it is required that the research efforts of each of us are recorded clearly, accurately and with necessary details so that the next research worker knows what his (or her) efforts are yielding. In this paper we have provided the details to set the records right. A summary of our results is presented in Table-16.

\begin{tabular}{|r|r|r|l|r|r|r|r|}
\hline \multicolumn{1}{|c|}{ Table-16. Minimum Values of } \\
Keane's Function of Different Dimensions Obtained in this Study \\
\hline Dim & Min[f(x)] & $\operatorname{Dim}$ & Min[f(x)] & Dim & Min[f(x)] & Dim & Min[f(x)] \\
\hline 2 & -0.36498 & 17 & -0.79151 & 30 & -0.81806 & 47 & -0.83330 \\
\hline 5 & -0.63445 & 20 & -0.80362 & 35 & -0.82528 & 50 & -0.83499 \\
\hline 10 & -0.74731 & 23 & -0.81004 & 40 & -0.82662 & 60 & -0.83775 \\
\hline 15 & -0.78165 & 27 & -0.81694 & 43 & -0.83226 & 100 & -0.84422 \\
\hline
\end{tabular}

We also conjectured that values of the decision variables diminish with the increasing index, that is, $x_{i}>x_{j} ; \forall j>i$ and

they form two distinct clusters with almost equal number of members. These regularities indicate whether the function could attain a minimum or (at least) has reached close to the minimum. They may also be exploited to seek the minimum values of the function. The Differential Evolution optimization program (developed by us) has gone a long way 
to obtain the optimum (if so!) results. Application of the Particle Swarm optimization program (developed by us) has clearly failed to minimize Keane's function of any considerable size, without arranging the variable values in a descending order. On ordering the variable values, the RPS results are comparable with those of the DE. The $\mathrm{DE}$ also needed ordering of variable values to perform when the size of the problem was larger. Our two findings are notable: (i) Keane's envisaged $\min (\mathrm{f})=-0.835$ for 50 dimensional problem is realizable by the RPS as well as the DE; (ii) Liu-Lewis' $\min (\mathrm{f})=$ -0.84421 for 200 -dimensional problem is grossly sub-optimal.

Finally, although Keane disfavors ordering of variables to obtain a minimum (since such 'tricks' lead to over-estimation of the efficiency of an optimizing program), such a trick, nonetheless, gives us new lower bounds of the function that might stimulate further improvements in different optimization procedures. We hold that our investigation should have two objectives: (a) obtaining the most efficient optimizers; (b) obtaining new lower bounds of the function for different dimensions.

\section{References}

- Emmerich, MTM: Single- and Multi-objective Evolutionary Design Optimization Assisted by Gaussian Random Field Metamodels, Dissertation for Doctoral Degree in Natural Sciences, University of Dortmund, Dortmund. 2005.

- Hacker, KA, Eddy, J and Lewis, KE: "Efficient Global Optimization using Hybrid Genetic Algorithms," in $9^{\text {th }}$ AIAA/ISSMO Symposium on Multidisciplinary Analysis and Optimization, Atlanta, Georgia, 4-6 September 2002.

- Keane, AJ: "Bump: A Hard(?) Problem” http://www.soton.ac.uk/ ajk/bump.html, 1994.

- Keane, AJ: "Experiences with optimizers in structural design," in Parmee, IC (ed) Proceedings of the 1st Conf. on Adaptive Computing in Engineering Design and Control, University of Plymouth, UK, pp. 14-27, 1994.

- Liu, P and Lewis, MJ: "Communication Aspects of an Asynchronous Parallel Evolutionary Algorithm", Proceedings of the Third International Conference on Communications in Computing (CIC 2002), pp. 190-195, Las Vegas, NV, June 24-27, 2002. http://grid.cs.binghamton.edu/papers/LiuAPEAComm_CIC.pdf

- Mishra, SK (a): "Global Optimization by Particle Swarm Method: A Fortran Program" (August 1, 2006). Available at SSRN: http://ssrn.com/abstract=921504

- Mishra, SK (b): "Global Optimization by Differential Evolution and Particle Swarm Methods: Evaluation on Some Benchmark Functions" (September 30, 2006). Available at SSRN: http://ssrn.com/abstract=933827

- Mishra, SK (c): "Performance of Differential Evolution and Particle Swarm Methods on Some Relatively Harder Multi-Modal Benchmark Functions" (October 13, 2006). Available at SSRN: http://ssrn.com/abstract=937147

- Ong, YS and Keane, AJ: "Meta-Lamarckian Learning in Memetic Algorithms" http://ntu-cg.ntu.edu.sg/ysong/journal/IEEE_EC_Ysong2003.pdf, 2003.

- Ong, YS, Lim, MH, Zhu, N and Wong, KW: "Classification of Adaptive Memetic Algorithms: A Comparative Study", Un-dated (possibly written in 2005) working paper, http://ntu-cg.ntu.edu.sg/ysong/journal/AdaptiveMA.pdf

*Contact: mishrasknehu@yahoo.com 